OPEN

\author{
SUBJECT AREAS: \\ APPLIED PHYSICS
}

GEOPHYSICS

CIVIL ENGINEERING

Received

10 December 2013

Accepted

1 May 2014

Published

27 May 2014

Correspondence and requests for materials should be addressed to H.O.G. (h.o.ghaffari@ gmail.com)

\section{Faulting of Rocks in a Three-Dimensional Stress Field by Micro-Anticracks}

\author{
H. O. Ghaffari, M. H. B. Nasseri \& R. Paul Young
}

Department of Civil Engineering and Lassonde Institute, University of Toronto, Toronto, 170 College Street, M5S3E3, ON, Canada.

Nucleation and propagation of a shear fault is known to be the result of interaction and coalescence of many microcracks. Yet the character and rate of the microcracks' interactions, and their dependence on the three-dimensional stress state are poorly understood. Here we investigate formation of microcracks during sandstone faulting under 3D-polyaxial stress fields by analyzing multi-stationary acoustic waveforms. We show that in a true three-dimensional stress state (a) faulting forms in a orthorhombic pattern, and (b) the emitted acoustic waveforms from microcracking carry a shorter rapid slip phase. The later is associated with microcracking that dominantly develops parallel to the minimum stress direction. Our results imply that due to inducing the micro-anticracks, the three-dimensional (3D) stress state can quicken dynamic weakening and rupture propagation by a factor of two relatively to simpler stress states. The results suggest a new nucleation mechanism of 3D-faulting with implications for earthquakes' instabilities, as well as the understanding of avalanches associated with dislocations.

T he mechanisms of rock faulting were mainly addressed by two macro-mechanical models, namely andersonian $^{1}$ and slip models ${ }^{2}$ that are compatible with field and experimental observations ${ }^{3-5}$. While the classic andersonian model (based on the Coulomb-Mohr criterion) predicts bimodal faulting (conjugate set of two faults), the slip model explains the polymodal fault patterns (orthorhombic sets of four faults) ${ }^{2,6,7}$, observed under a true triaxial stress state $\left(\sigma_{1}>\sigma_{2}>\sigma_{3}\right)$. From a micro-mechanical point of view, based on acoustic emission patterns in conventional triaxial tests (i.e., $\sigma_{1}>\sigma_{2}=\sigma_{3}$ ), the shear faulting is related to the interaction and coalescence of brittle, dilational microcracks ${ }^{8-10}$. The prevailing observation is that these microcracks are preferentially aligned parallel to $\sigma_{1}-\sigma_{2}$ plane, namely, normal to the least compressive stress ${ }^{6,8,10}$. On the other hand, the microcracks that are associated with deep-focus earthquakes, which occur under high pressure and high temperatures, are possibly oriented perpendicular to maximum compressive stress, and termed anticracks ${ }^{11,12}$. An important feature of deep-earthquakes, which may be related to their anticrack source, is that they start up significantly faster (i.e., shorter rise time) than do intermediate or shallow depth earthquakes ${ }^{13,14}$. Here, we show that shorter rising time is not restricted to deep-focus earthquakes, and is observed in unstable slip under a truetriaxial stress state. We monitored the detailed time evolution of 3D faulting that led to polymodal, complex fault systems, and visualize this dynamic evolution of rupture-forming faults under true-triaxial test conditions (TTT). We found that the nature of unstable slip under TTT can be different from unstable slip under conventional triaxial tests (CTTs). We show that fault-nucleation in TTTs is associated with shorter generic phases of emitted waveforms which imprints unique signatures in appropriate phase spaces, and they differ from rupture fronts in CTTs. Our experiments further highlight a potential new weakening mechanism which implies that micro faults reach about a factor of two faster to steady state frictional strength.

\section{Results}

Our experimental system is schematically shown in Fig. 1a. A cubic sample of Fontainebleau sandstone $(80 \times 80$ $\times 80 \mathrm{~mm}$ ) with 3 acoustic emission (AE) transducers per face is loaded normal to its faces. The stresses $\sigma_{2}=$ $35 \mathrm{MPa}$ and $\sigma_{3}=5 \mathrm{MPa}$ are maintained constant during the experiment (see section 1 and 4 of supplementary materials for other test types). The superimposed best-located AE events on the reconstructed micro X-ray patterns showed that the evolution of faulting follows "diffusive" fracture patterns, forming the final shape of fault patches (Figs. 1c, d, S1). This is a typical example of composite self-organized cracks, observed frequently in cylindrical CTTs ${ }^{15,16}$. Despite this similarity, we found that the evolution of acoustic signals corresponding to rupture fronts is different in TTTs.

To explore the above observations, we used network theory modeling on the recorded multi-stationary acoustic signals ([17]). We use three parameters of this theory to characterize the AE distribution and evolution. The Q 


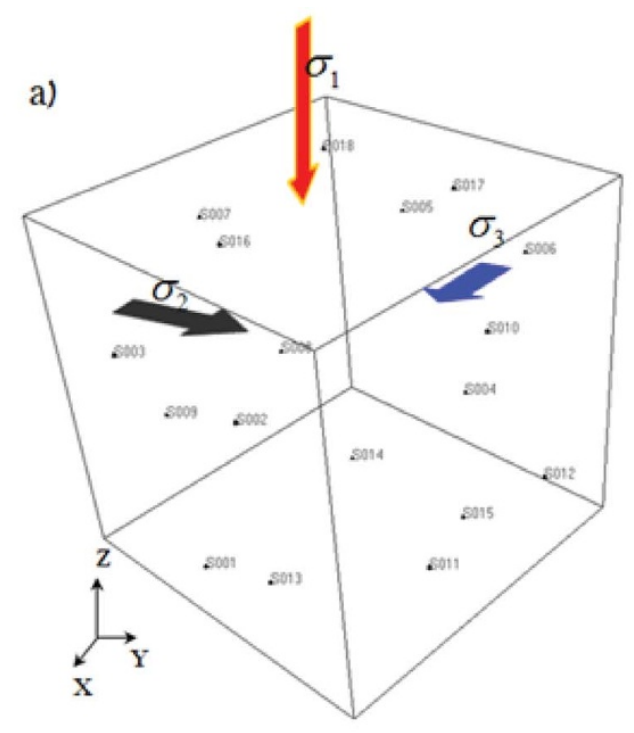

c)

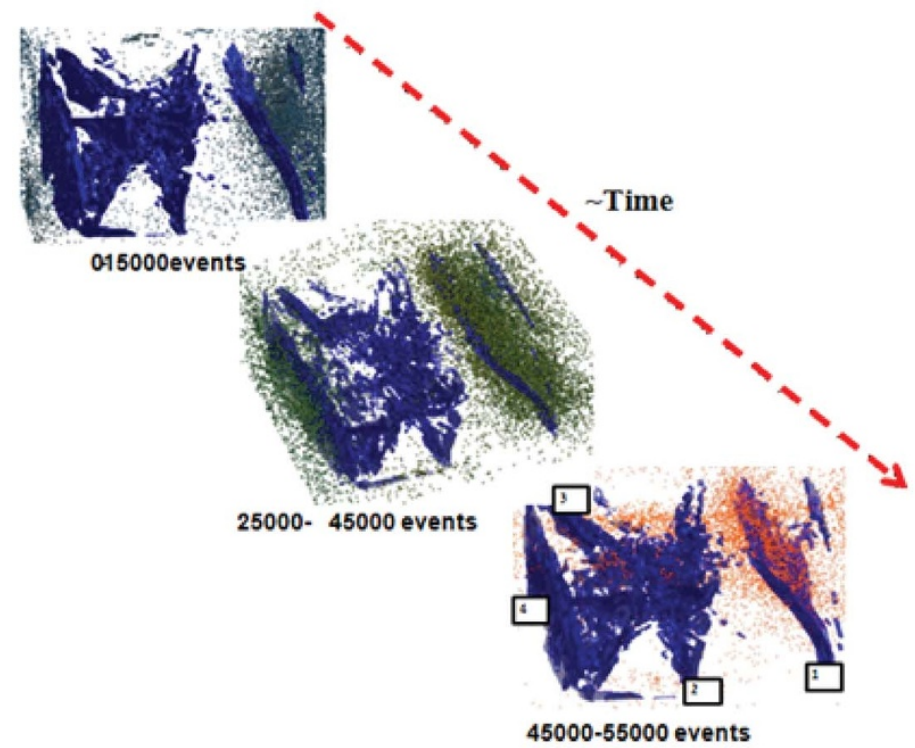

b)

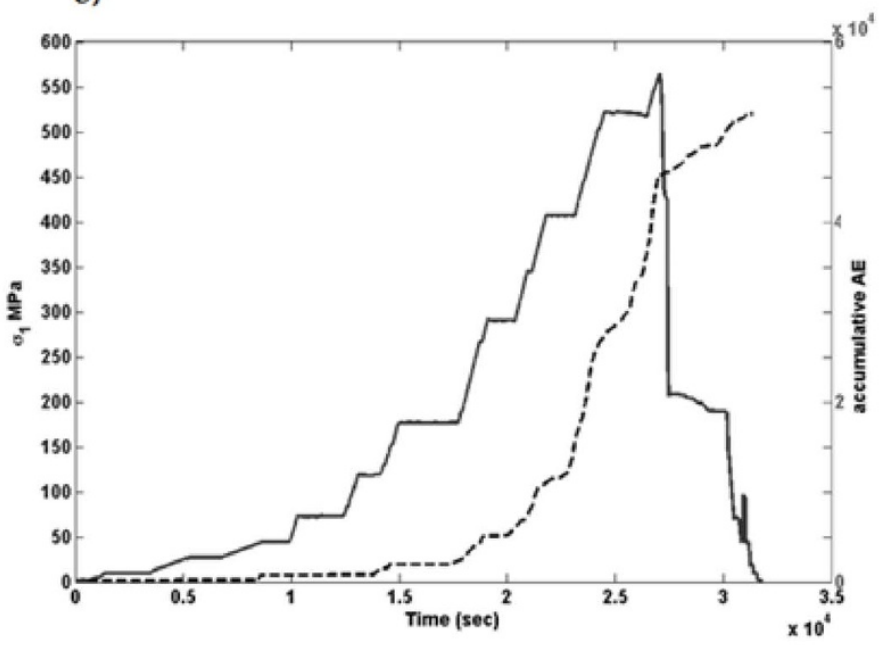

d)

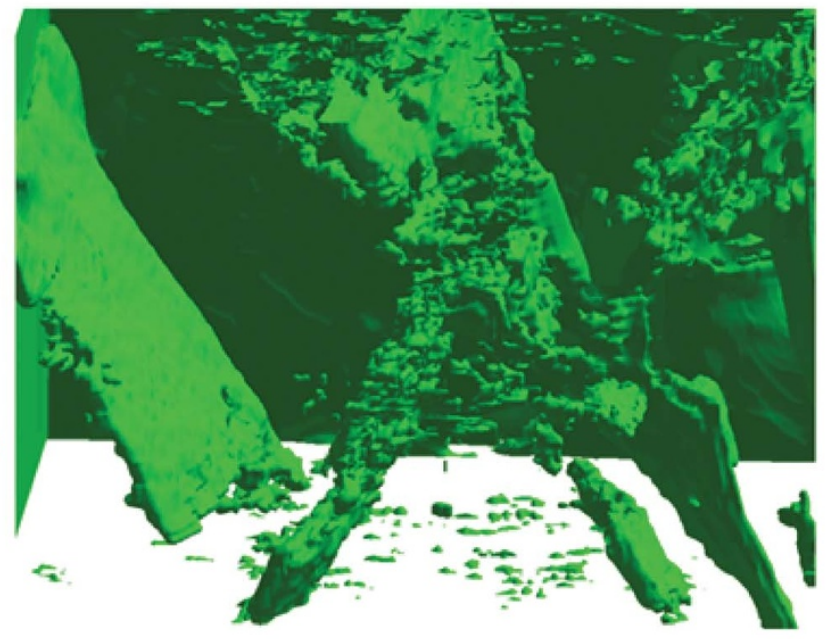

Figure 1 Study of Orthorhombic faulting under the true triaxial tests. (a) a schematic of the experiment procedure and remote stress field configuration have been shown where the numbers on each face of the cube shows the piezoelectric transducers. (b) The evolution of the main driving stress field $\left(\sigma_{1}\right)$ and accumulated recorded acoustic emissions during a few hours of the experiment. The stairlike trend of the loading curve is due to pausing the test in order to measure permeability in three directions. (c,d) Superimposed events on the best-located events superimposed on the reconstructed fault patterns from X-ray images and the temporal/spatial evolution of the events (i.e., event-sets' sequences). The first two event-sets show the slow growth of the external fault-system's patches while the fast-growth of the internal branches of the Orthorhombic faulting system is observed around the main stress drop (Fig.S1). The numbers shows 4 main fault sets. (d) A close view of 3D - fault system.

parameter is the 'modularity' of the network that characterizes the number of modules of network of the AE event. The B.C parameter is defined as the "betweeness centrality" that approximately is correlated with the magnitude of the AE events. The lambda parameter is defined as the maximum eigenvalue of Laplacian of the network (see Methods part for definition and details), which scales with the inverse of Q parameter in our experiments. Applying these parameters on acoustic signals, we found three generic time scales, corresponding to the following phases in Q-profiles or modularity of corresponding functional networks ${ }^{17}$ : (1) a nucleation and main deformation phase in the form of initial strengthening (2) a fast-slip or weakening phase, and (3) a slow slip or decelerating stage (Fig. 2a). Comparing the evolutionary phases of micro-cracks to dynamic stress change from macro-sliding events (recorded by strain gauges) reveals similar time characteristics and trends (Fig. 2c, d). In using the reciprocal of Q-profiles, we magnify the initial strengthening phase (we call it R-profile) to obtain the maximum strength (Fig. 2b).

We found (SI, Figs. S2) that the duration of the fast-slipping phase of precursor events does not effectively change under a wide range of loading conditions including different strain rates, acoustic signal feed-back control test, smooth and rough fault frictional interface with 150 and $200 \mathrm{MPa}$ confinement pressures (Westerly granite samples), and double-direct shear test with quartz-gouge materials. These observations strongly indicate that the fast-slip phase is a generic phase in micro-cracks, which is not sensitive to variations of loading conditions in CTT or conventional friction tests. We note that a nearly invariant time scale of the second phase has been recently reported ${ }^{18}$ on engineering polymeric materials (and interfaces) and has been shown to be insensitive to the dimension of loaded samples. Comparing the fast-slip times of micro-cracks under 
a)
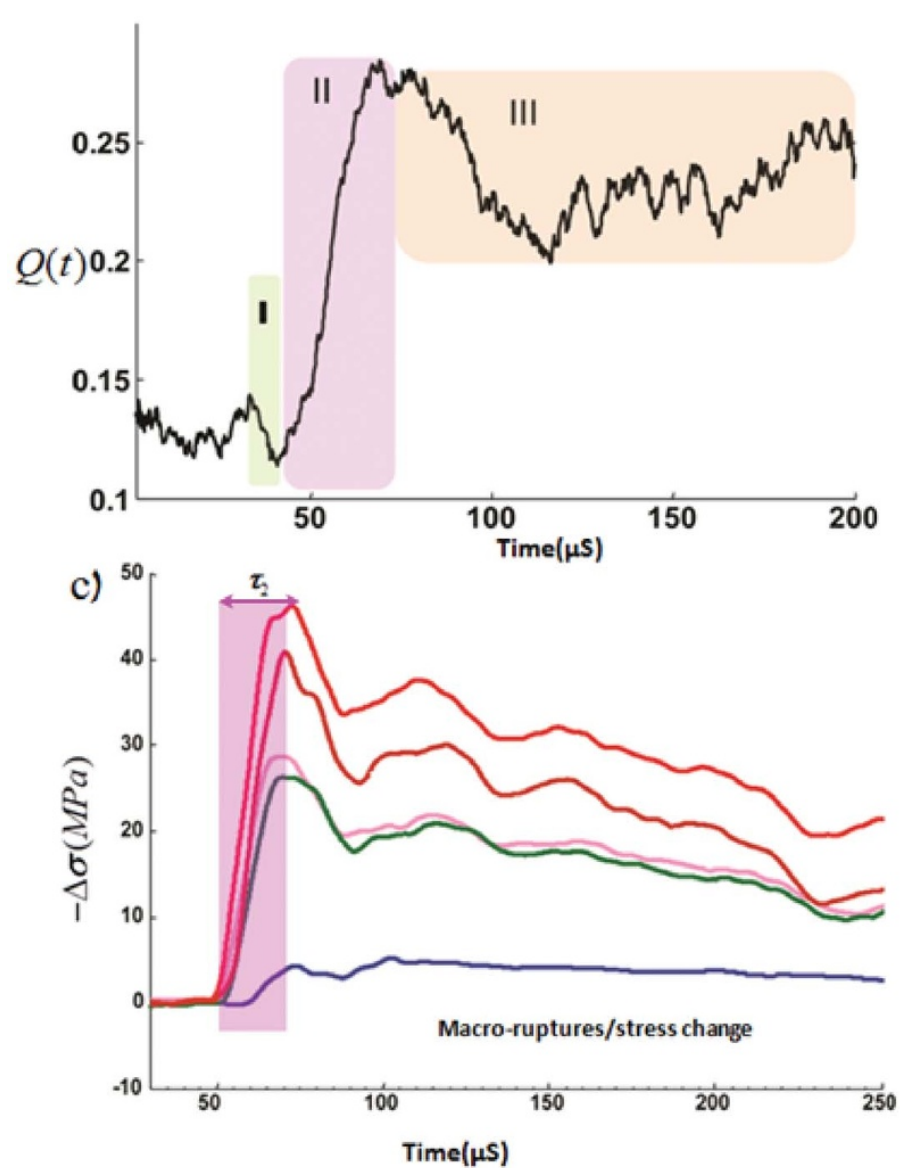

b)
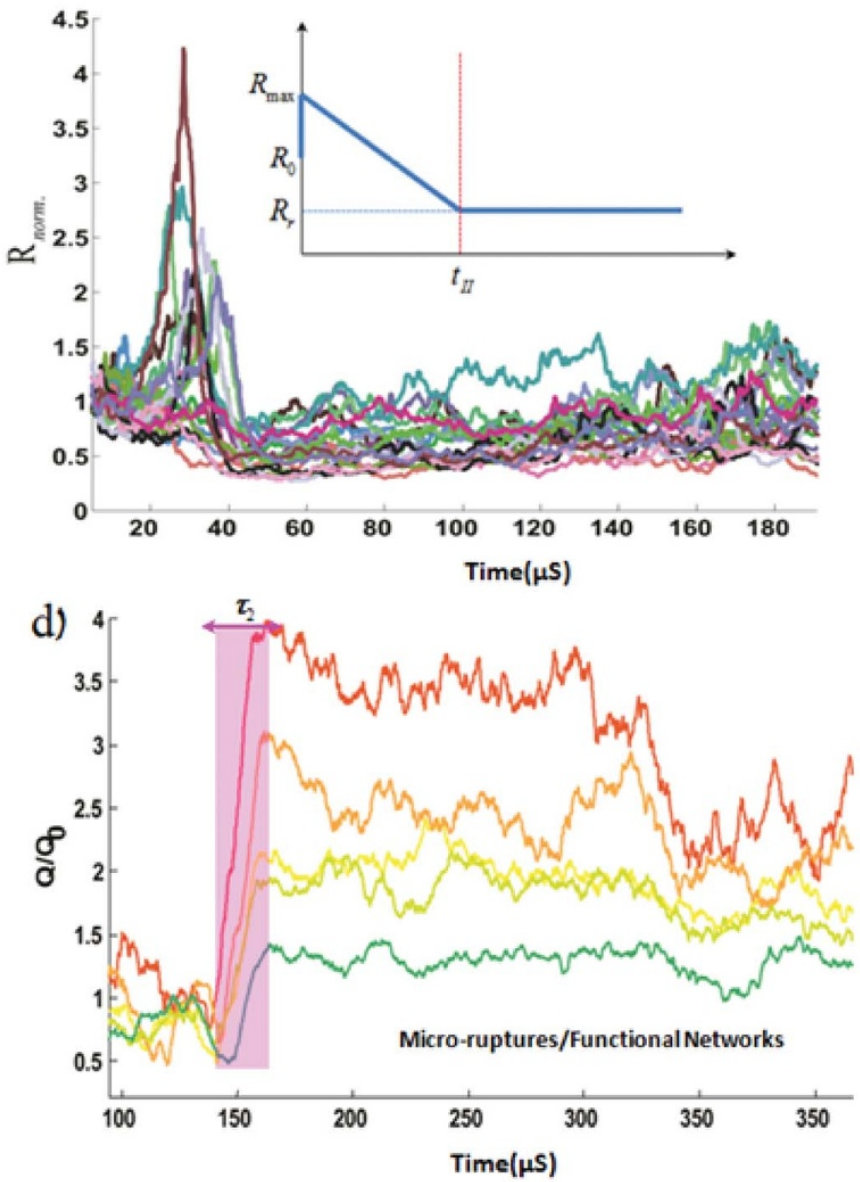

Figure 2 Q-profiles resemble dynamic stress changes in micro-scale failures. (a) The main phases of Q-profiles includes three main stages: (1) initial strengthening, (2)fast-slip or weakening phase and (3) deescalating or slow-slip phase. The profile is from CTT- westerly granite experiment ${ }^{16}$. (b) The maximum dynamic strength is inferred from R-profile as the inverse of Q-profile which places emphasis on the evolution of the first phase. We have shown $\sim 20$ acoustic excitations from TTT (FTB4). The inset illustrates a simplified R-profile. The weakening stage can be described by the timeweakening model which prescribes that strength of heterogeneity weakens during a characteristic time $\left(\boldsymbol{\tau}_{I I}\right)$. (c, d) Dynamic stress changes from 5-macro stick-slip (Saw-cut fault evolution ${ }^{16,17}$ in Westerly Granite) resemble similar evolutionary phases and time-characteristics with Q-profiles in microruptures $(\mathrm{d})$.

CTT, from different rocks (with dominant silicate minerals), concrete samples(Portland cement), thermally treated Westerly granite specimens $\left(850^{\circ} \mathrm{C}\right.$ and $\left.250^{\circ} \mathrm{C}\right)$, Polymers and steel alloy implied that this generic phase is linked to the ductility of materials, and changes in energy dissipation mechanisms could alter the duration of the second phase (section3 of SI and Fig. S.3). For instance, this period is longer for concrete -as cement based materials-due to the cement component and Calcium-Silicate-Hydrate (C-S-H) structures (Fig.S.3c). This is due to additional sources of energy dissipation rather than the creation of new surfaces ${ }^{19}$. For the same reason thermally treated $\left(850^{\circ} \mathrm{C}\right)$ westerly granite specimens, characterized by much higher intergranular and intragranular crack density than $250^{\circ} \mathrm{C}$ specimens, -shows a fast-slip time of $\sim 40 \mu$ s in comparison to the later specimen $(\sim 20 \mu \mathrm{s})$. Also we discover that this phase is about $\sim 70 \mu$ s for Poly (tetrafluoroethene), close to $\sim 60 \mu$ s for $\mathrm{PMMA}^{18}$. This finding suggests that longer fast-slip phase likely indicates a signature of plasticity in micro-cracks (i.e., significant ductile cracking).

Interestingly, examining the same interval for a true triaxial test shows a shorter time interval $(\sim 10 \mu \mathrm{s})$ for the same sandstone (Fig. 3a (and inset), b). We have observed the mentioned phenomenon from different events, occurring in different times and positions.
This observation implies a different pulse shape for acoustic-cracking noises under 3D boundary conditions while a universal left-asymmetric shape of the pulse is maintained for both CTT and TTT (section3 of SI). To further analyze the shorter fast-slip phase, we use a simple effective temperature model $([18,20]$-section 2 of supplementary materials). The model estimates that the cracks under the triaxial stress field carry a smaller amount of energy (at least $40 \%$ of CTT), indicating different nature of cracking. We support the idea of micro anti-cracks with observation of significant activity of microcracks in the direction of the minimum stress state, forming anitcracks (Fig. 3c-sections 4,5 of SI - Fig.S.1,S.6, S.18-19). We speculate that anti-cracks form rupture fronts, induced by far field stress state, results in a faster slipping or weakening period.

To see whether micro-anticrackings changes the shape of waveforms, we examined typical waveforms during the formation of anticracks in a multi-anvil test (High pressure -High temperature: HP-HT) on the Olivine samples (section 5 of SI - Fig.S23-S24), which revealed $\sim 8 \mu$ s of the fast-slip phase. Thus, this observation of events from the HP-HT experiment confirms the shorter rising time of natural deep-focus earthquakes ${ }^{13,14}$; a feature that is similar to the shorter weakening phase of rupture fronts in TTTs. Interactions of such anticracks with each other likely form the final fault surface 
a)

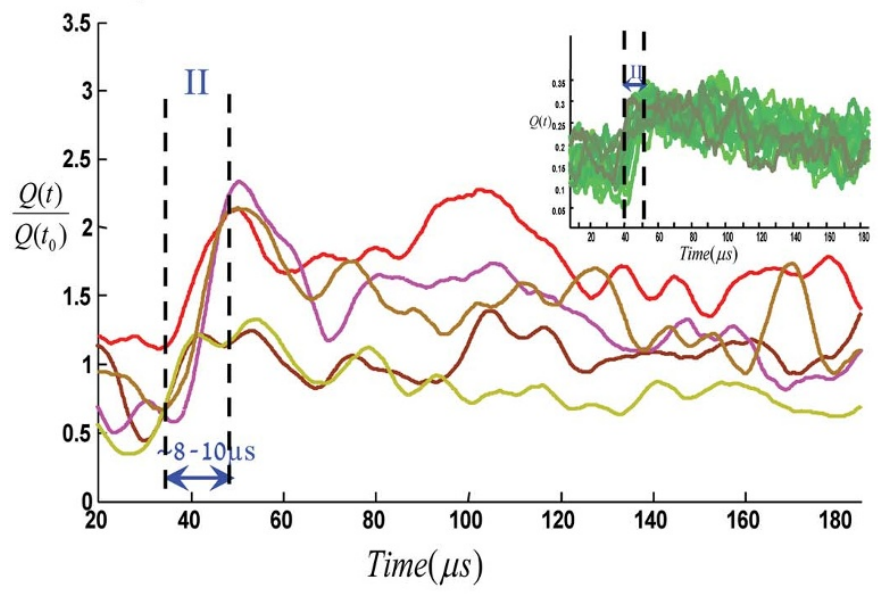

b)

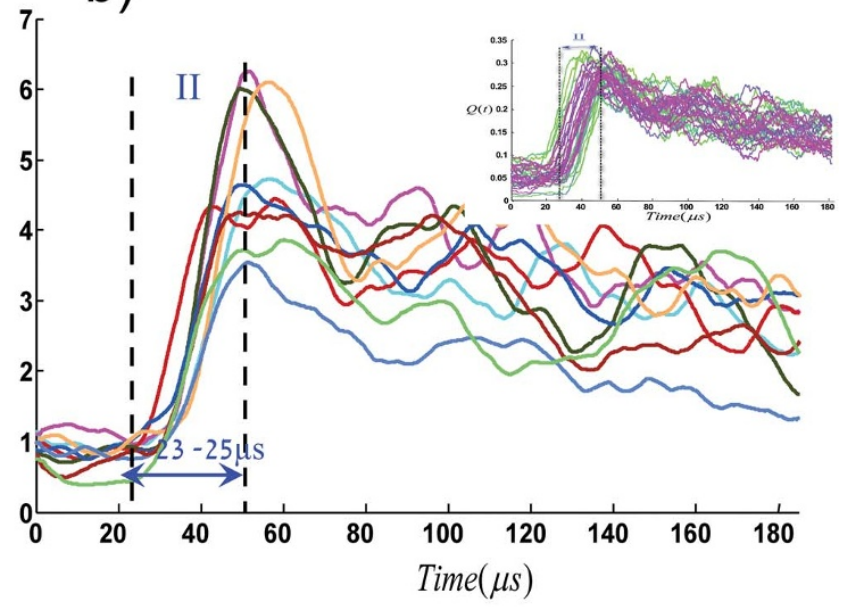

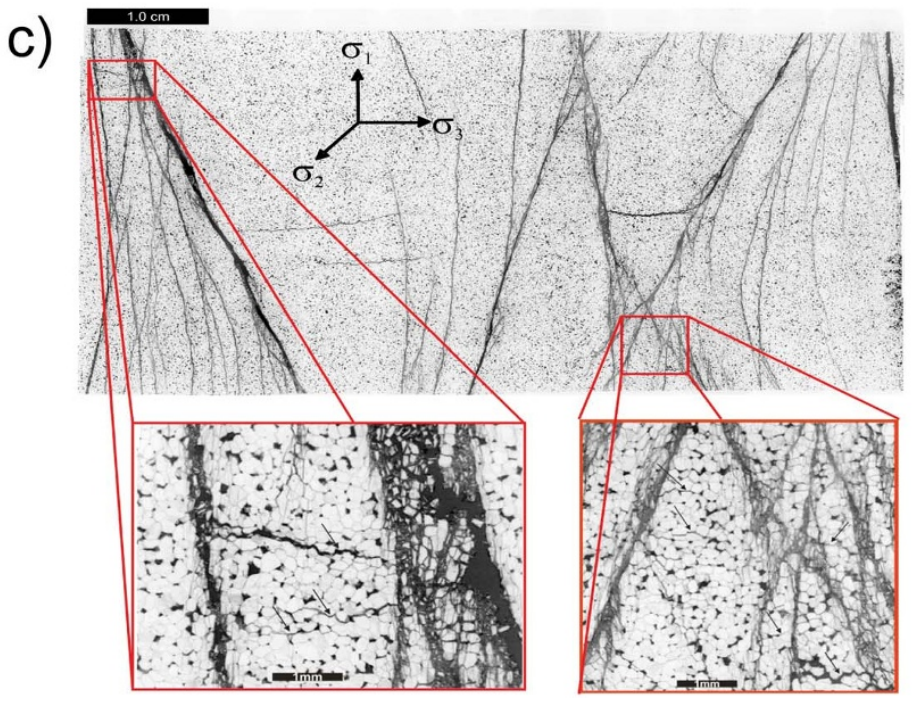

Figure 3 Q-profiles and fast-slip phase in TTT and CTT. (a) Q-profiles from a TTT test (FTB4) show an $\sim 8-10 \mu$ s duration of the fast-slipping phase (inset: 150 events from TTT-FTB2; see section 4 in the supplementary information). (b)The same sandstone under the conventional test shows a longer fast slip phase $(\sim 23-25 \mu \mathrm{s})$. Inset shows 45 events from the CTT. $Q\left(t_{0}\right)$ is the initial or rest value of modularity. (c) Visible (anti-) cracks parallel to minimum stress field in TTT.

which can be explained with a microcrack interaction model such as the three-dimensional solution of Eshelby ${ }^{6}$. The concept of anticracks previously has been also used to analyze the failure process in high porosity sandstone as a theory of compaction bands ${ }^{21}$, to characterize the triggering mechanism of snow avalanches ${ }^{22}$ as well as the localized dissolution of limestone ${ }^{23}$. We conclude that anticrack form of rupture fronts and possible substantially lower fracture surface energy are the main mechanisms of our observations. Due to the frequent observation of micro-faults with dominant double couple source mechanism (i.e., shear component), we propose that true $3 \mathrm{D}$ stress states can induce rapid weakening on 3D-faults, faster than conventional weakening mechanism(s). This fast-release of energy is associated with fast-acceleration ${ }^{24}$ which shortens the slip-weakening distance. The nature of this slip-acceleration is different from one demonstrated $i^{24}$ which is associated with fault wearing and gouge formation; here we assign the quickened phase to grow of microanticracks.

Furthermore, we show that network parameter spaces of TTTs' events, collectively, are different from CTTs' events. Considering mean-temporal of maximum Laplacian $\left(\bar{\lambda}_{\max }\right)$ and spatio-temporal mean of centrality $(\overline{\log <B . C>})$ on networks (see Methods part for definition), we find that recorded events from TTTs and CTTs collapse in $\bar{\lambda}_{\max }-\overline{\log \langle B . C\rangle}^{-1}$ plane (Fig. 4a), which confirms recent similar observations from the rock-frictional interfaces ([17]Fig.S8,Fig.S.14 and Fig.S.17). Considering that $\bar{Q} \propto{\overline{\lambda_{\max }}}^{-1}$ and $\bar{Q} \propto \bar{\sigma}^{-1}$ ( $\bar{\sigma}$ is the temporal average of stress change during the monitoring interval), we deduce that events with higher $\bar{\lambda}_{\max }$ indicate ruptures with higher weakening rate and then high rupture velocity ${ }^{34}$. As we have shown in Fig. 4a-inset, the conventional tests imprint three main clusters of events in $\bar{\lambda}_{\max }-\overline{\log \langle B . C\rangle}^{-1}$, corresponding to the energy spectrum of ruptures ${ }^{17}$. Comparing

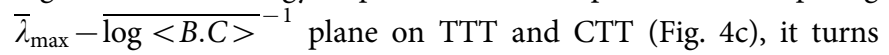
out that in TTT, we cannot recognize distinctive clusters, indicating that probability of finding "abnormal" events in terms of the rupture's speed regimes under true triaxial conditions is rare. Thus, these observations imply that energy of events is more localized in TTTs. This indicates that the shapes of waveforms are also bounded rather than CTT cracking noises (Fig.S.15-18).

To complete our analysis, we speculate that the widely discussed failure criterion of materials under TTTs ${ }^{2,25-27}$ can be inspected in the scale of tiny-amplified events (i.e., micro-failure criterion). To 
a)

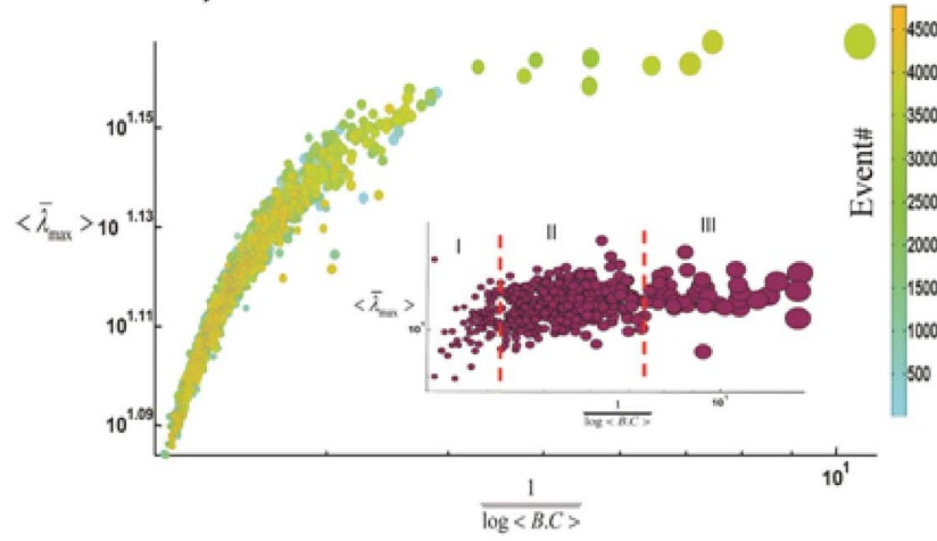

b)

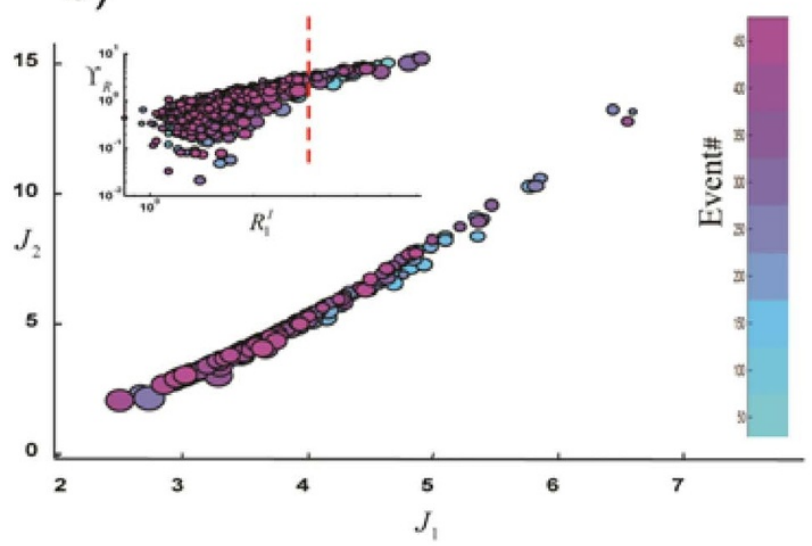

c)

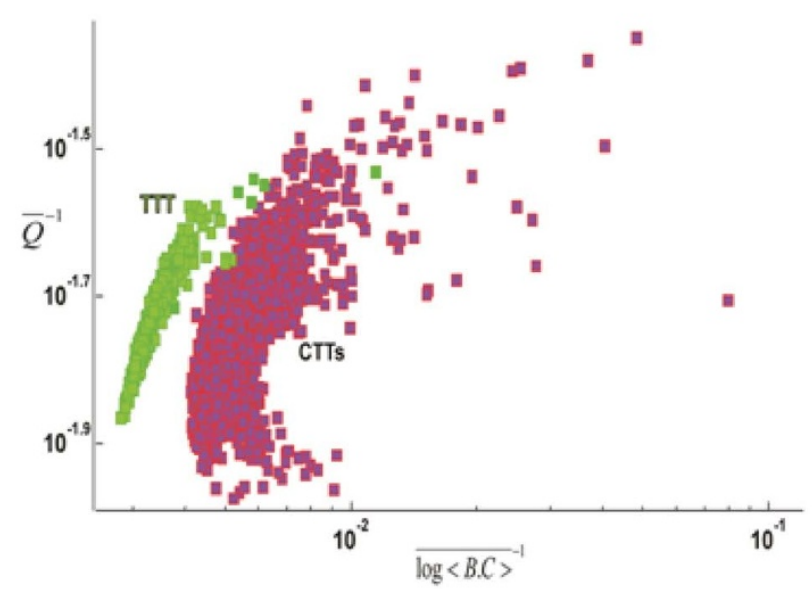

Figure $4 \mid$ Global and local networks' parameter spaces from precursors failures on TTT and CTT. (a) $\bar{\lambda}_{\max }-\overline{\log }<B . C>^{-1}$ parameter space on $\sim 4500$ events (including weak events) an hour prior to the main-final faulting development in FTB4 (section 3 of SI). Inset shows events from the CTT at the same plane with three main classified classes corresponding to three classes of rupture fronts energies ${ }^{17}$. Class I, II and III corresponds with slow, regular and very fast weakening rates, respectively. Generally $\bar{\lambda}_{\max } \propto \bar{Q}^{-1}$ and $\bar{Q} \propto \bar{\sigma}^{-1}$ (b) a study of independent layers of networks. The sub-network's parameter space: collapsing $\sim 520$ strong events from the FTB4 test in the $J_{1}-J_{2}$ parameter space, where $J_{1}=R_{1}^{I}+R_{2}^{I}+R_{3}^{I}$ and $J_{2}=R_{1}^{I} R_{2}^{I}+R_{2}^{I} R_{3}^{I}+R_{1}^{I} R_{3}^{I}$. A power law relation satisfies an excellent collapsing of events in the $J_{1}-J_{2}$ phase diagram as: $J_{2} \propto J_{1}^{b}, b \approx 2.6$. This scaling confirms Reches' proposed failure criterion $^{2}$. In the inset, we have shown the $R_{1}^{I}-\Upsilon_{R}$ plane with $\Upsilon_{R}=\sqrt{\left(R_{1}^{I}-R_{2}^{I}\right)^{2}+\left(R_{2}^{I}-R_{3}^{I}\right)^{2}+\left(R_{3}^{I}-R_{1}^{I}\right)^{2}}$-proportional to $\tau_{\text {oct. }}$ - for the same events (Also see Fig.S.5-Fig.S.15). (c) a collection of $\sim 3000$ events from CTTs and TTTs (see the list of CTTs in SI) in the normalized $\bar{Q}^{-1}-\overline{\log }<B . C>^{-1}$ map.

envisage such micro-failure criterion, we consider the possible relation between independent layers of functional multiplex acoustic networks (Methods part), in which an independent network of sensors is assigned for each main direction $(\mathrm{X}, \mathrm{Y}$ and $\mathrm{Z})$. Then, for each occurred rupture front and for each sub-network (layer), we calculate the reciprocal of the minimum modularity index, namely "maximum resistivity" ( $R_{i}^{I}$ indicates the maximum resistance against motion from $i^{\text {th }}$ layer or sub-network in the first phase of Q-profiles Fig.S7 and Fig.S9-S17). Since $R_{i}^{I}$ occurs in the main deformation phase of Q-profiles (i.e., first stage), then it is proportional with the maximum strength in that direction $\left(R_{i}^{I} \propto \sigma_{\max }^{i \in\{X, Y, Z\}}\right)$. Remarkably, the events forming the Polymodal fault-system collapse in the $J_{1}-J_{2}$ parameter space in which $J_{1}=R_{1}^{I}+R_{2}^{I}+R_{3}^{I}$ and $J_{2}=R_{1}^{I} R_{2}^{I}+R_{2}^{I} R_{3}^{I}+$ $R_{1}^{I} R_{3}^{I}$ (Fig. 4b), represent an excellent power-law: $J_{2} \propto J_{1}^{b}, b \approx 2.6$ (also see section 3 in SI). Indeed, $J_{1}$ and $J_{2}$ are proportional with the stress invariants where $J_{1} \propto \sigma_{1}+\sigma_{2}+\sigma_{3}$ and $J_{2} \propto \sigma_{1} \sigma_{2}+\sigma_{2} \sigma_{3}+\sigma_{1} \sigma_{3}$. Interestingly, the obtained power-law and similar relations (Fig. 3binset) on other parameters match with the macro-failure criterion presented by Reches ${ }^{2}$, Mogi $^{25}$ and Haimson ${ }^{26}$, indicating that possibly a similar mechanism governs both precursor and final failures (i.e., significant drop of the driving stress).

In addition, based on Mogi's approach ${ }^{25}$, under TTTs, the ductility of rocks decreases. To show the validation of this character in microfailures, we note that the signature of ductility (plasticity) is encoded in weakening time (SI, Fig.S3), so that it generally increases the duration of this phase which leads to the further decrease of $\bar{\lambda}_{\max }$ and the possible formation of distinct cluster. Then, due to the absence of the lower cluster (cluster I in Fig. 4a-inset; Fig. 4c) in $\bar{\lambda}_{\text {max }}-{\overline{\log \langle B . C\rangle^{-1}}}^{-1}$ from TTTs, we speculate that a similar approach holds in micro-failures. The same logic can be followed for $\sigma_{1}-\tau_{\text {oct. }}$ plane which is proportional with $R_{1}^{I}-\Upsilon_{R}$ (Fig. $4 \mathrm{~b}$-inset) in which $\tau_{\text {oct. }}=\frac{1}{3}\left(\left(\sigma_{1}-\sigma_{2}\right)^{2}+\left(\sigma_{2}-\sigma_{3}\right)^{2}+\left(\sigma_{1}-\sigma_{3}\right)^{2}\right)^{1 / 2}$. In other words, we have shown that the long-standing problem of failure criterion in a 3D stress field has its origin in each single recorded precursor event (i.e., local failure). 


\section{Discussion}

Our results have significant implications for earthquake seismology, rock-mass stability and fluid migration and 3D permeability anisotropy in fractured rocks. Our results provide a link to prove that accommodation of $3 \mathrm{D}$ strain due to polymodal fracture patterns change strength (encoded in failure criterion). For the first time, we showed that micro-anticracking can change slip-weakening rate and accelerate the rate of energy release. This implies a sharper source time function for further modeling of our experiments. Indeed, our results can be assumed as an extension to detachment fronts in micro-faults ${ }^{18}$ to a general concept of anti rupture fronts. In addition, we showed that deep earthquakes and fault instabilities under 3D-polyaxial stress fields diverge little in terms of the stress drop invariance (i.e., fast-slip duration), promoting the recent approach on similarity of deep earthquakes to their shallow counterparts ${ }^{33}$. However, this similarity does not imply that the same physics governs both weakening courses. In deep-focus earthquakes, it is likely the nucleation is associated with dislocation density and complex motion of dislocations is encoded in fast-slip regime (Fig.S.24) ${ }^{35}$. Regarding nucleation of anti-cracks in our TTTs, since the grains of sandstone can arrange in a tightly packed configuration, the volumetric collapse and cohesion along the crack faces significantly shorten the critical displacement ${ }^{22}$. Our results confirm that the growth of anti-cracks (as the source of true compaction bands) is due to rapid propagation as suggested $\mathrm{in}^{21}$. From another perspective, studies in atomic friction anisotropy revealed differences in acoustic excitations can be related to different crystallographic directions where in a certain direction the energy dissipation is minimum ${ }^{36}$. Our findings using networking of anti micro-cracking's acoustic excitations in TTTs coincides with such observations. The presented results might be applicable for emitted events from dislocations in atomic scales which radiate elastic energy in terms of acoustic emissions $^{28}$. In terms of the analysis of micro damages, our algorithms provide unique tools to understand the details and physics of multistationary acoustic waveforms. In fact, these algorithms can be employed to extract further information on other sources of acoustic excitations. Developing models based on multiplex-functional networks and extending the proposed network analysis to natural earthquakes will be a future project.

\section{Methods}

Summary of experimental procedures. Cubic-samples of the saturated Fontainebleau sandstone are loaded at the University of Toronto, in a custom-made true triaxial cell ${ }^{29}$. The triaxial cell can be used to measure permeability independently in different directions under raised temperatures. Special attention was paid to keep the balance of loads in parallel directions to avoid possible torque. The strain rate for all axial directions was $3 \times 10^{-6} \mathrm{~s}^{-1}$. The waveforms were recorded using 18 piezoelectric transducers at a $10 \mathrm{MHz}$ sampling rate while triggered sensors are employed to detect the events. Another system simultaneously and continuously recorded all events from all transducers during the aforementioned experiments. For conventional compressive tests, we used the results of previously reported experiments $^{30}$ while the samples were saturated and the events were chosen from precursor rupture fronts. A Fontainebleau sandstone specimen (length $=88 \mathrm{~mm}$, diameter $=40 \mathrm{~mm}$ ) was deformed inside a triaxial. A network of piezoceramic transducers (PZT) was used in $10 \mathrm{MHz}$ sampling rate with similar criterion to the Polyaxial test to detect the events. In the supplementary document (section1 and 5), we have -also-included the summary of other tests (and their multiplex networks attributes) on Westerly Granite samples under different loading conditions and geometry as well as smooth and rough rock-frictional interfaces ${ }^{16,17}$. Moreover, we used our ultrasonic imaging triaxial cell to record the events emitted during testing of $850^{\circ} \mathrm{C}$ and $250^{\circ} \mathrm{C}$ thermally treated Westerly granite specimens $(125 \mathrm{~mm}$ long and $50 \mathrm{~mm}$ in diameter) tested under $25 \mathrm{MPa}$ of confining pressure. In addition, we analysed multi-stationary waveforms from a Multi-anvil test (High pressure -High temperature tests) on olivine samples (section 5-SI).

Functional multiplex networks on acoustic emission waveforms. We define a multiplex network on the cubic geometry over acoustic transducers where it includes three main layers (the layers correspond to $\mathrm{X}, \mathrm{Y}$ and $\mathrm{Z}$ directions as the minimum, intermediate and maximum principle stress). To build networks, we use the algorithm introduced in ${ }^{17}$. We built a network on each time step in which the nodes correspond with acoustic sensors where elastic "excitement" induces voltagefluctuations in them. Please note that the "functional" friction networks are not structural networks; however underlying functionality of constructed networks correlates with main mechanical features of crack instabilities (see SI for more information and details of algorithms). Two cases were analyzed through this research: global networks or interdependent layers and local-sub networks as independent layers of mathematical graphs. Each node in functional networks was characterized by its degree $k_{i}$. For a given network with $N$ nodes, the degree of the node and Laplacian of the connectivity matrix were defined by

$$
k_{i}=\sum_{J=1}^{N} a_{i j} ; L_{i j}=a_{i j}-k_{i} \delta_{i j} \text { where } k_{i}, a_{i j}, L_{i j} \text { are the degree of } i \text { th node, elements of a }
$$

symmetric adjacency matrix, and the network Laplacian matrix, respectively. The eigenvalues $\Lambda_{\alpha}$ are given by $\sum_{J=1}^{N} L_{i j} \phi_{j}^{(\alpha)}=\Lambda_{\alpha} \phi_{i}^{(\alpha)}$, in which $\phi_{i}^{(\alpha)}$ is the $i$ th eigenvector of the Laplacian matrix $(\alpha=1, \ldots, N)$.We define $\lambda_{\max }=\left(-\Lambda_{N}\right)$ as the maximum eigenvalue of the Laplacian of the network. The betweenness centrality (B.C) of a node is defined as $^{31}$ :

$$
\text { B. } C_{i}=\frac{1}{(N-1)(N-2)} \sum_{\substack{h, j \\ h \neq j, h \neq i, j \neq i}}^{N} \frac{\rho_{h j}^{(i)}}{\rho_{h j}}
$$

in which $\rho_{h j}$ is the number of the shortest path between $h$ and $j$, and $\rho_{h j}^{(i)}$ is the number of the shortest path between $h$ and $j$ that passes $i$. The spatio-temporal average of B.C is indicated by $\overline{\log \langle B . C\rangle}$ where $\langle\ldots\rangle$ and bar-sign correspond to the spatial (i.e., nodes) and temporal averages, respectively. $\operatorname{In}^{17}$, we speculate that events from saw and rough fault cut-experiments flow in $\bar{\lambda}_{\max }-{\overline{\log \langle B . C\rangle^{-1}}}^{-1}$ plane with three distinguished trends, corresponding to rupture regime.

The Q value per each network (i.e., in each time-step) as the networks' modularity characteristic is given by ${ }^{31,32}$ :

$$
Q=\sum_{s=1}^{N_{M}}\left[\frac{l_{s}}{L}-\left(\frac{d_{s}}{2 L}\right)^{2}\right]
$$

in which $N_{M}$ is the number of modules (clusters), $L=\frac{1}{2} \sum_{i}^{N} k_{i}, l_{s}$ is the number of links in module and $d_{s}=\sum k_{i}^{s}$ (the sum of nodes degrees in module s). Using an

optimization algorithm (Louvain algorithm-[31]), the cluster with maximum modularity $(Q)$ is detected. The temporal evolution of $Q$ values in a few hundred microseconds evolution of waveforms provides Q-profiles.

As we have shown in Fig. 2.and Fig.S4, the main time characteristics of the evolutionary phases of Q-profiles and their trends from micro cracks coincides with dynamic stress profiles in macro-ruptures (a few centimeter scale). Then, we infer that the maximum $\mathrm{Q}(\mathrm{t})$ corresponds with the maximum stress drop $\left(\boldsymbol{\sigma}_{\mathrm{min}}\right)$ in submicron scales. The initial drop of Q-profile (as the nucleation and strengthening phase) before the weakening phase corresponds with maximum strength of heterogeneity (or asperity). The minimum value of $\mathrm{Q}(\mathrm{t})$ (or maximum value of $\mathrm{R}(\mathrm{t})$ ) coincides with $\sigma_{\text {max. }}$ Inferring this $\sigma_{\text {max. }}$ in each main directions (such as X-Y-Z) provides the ingredients to infer local failure criterion. For this reason, we address the maximum values of $\mathrm{R}(\mathrm{t})$ in three main directions in our TTTs. We also note that for all our data set, we have: $\bar{Q} \propto \frac{1}{\lambda_{\max }}$. Regarding interpretation of $\overline{\log }\langle B . C\rangle^{-1}$, we note that minimum value of $\log \langle$ max. $C>$ generally coincides with the large amplitudes of waveforms (Fig.S.6). Then, we infer rupture with high energy will have larger ${\overline{\log }\langle B . C\rangle^{-1}}^{-1}$ There are some indications that critical temporal curvatures of $\mathrm{Q}(\mathrm{t})$ represent significant fluctuation in $\overline{\log \langle B . C\rangle^{-1}}$ (Fig.S.5). With using the layers of multiplex networks and distribution of $\overline{\log \langle B . C\rangle^{-1}}$ in each layer (or direction), we can infer the tendency of rupture per each recorded micro-event (Fig.S.7).

1. Anderson, E. M. The Dynamics of Faulting and Dyke Formation with Applications to Britain (Oliver and Boyd, Edinburgh, 1942).

2. Reches, Z. Faulting of rocks in three-dimensional strain fields. II. Theoretical analysis. Tectonophysics. 95, 133-15 6 (1983).

3. Jones, R. R., Holdsworth, R. E., McCaffrey, Clegg, K. J. P. \& Tavarnelli, E. Scale dependence, strain compatibility and heterogeneity of three-dimensional deformation during mountain building: a discussion. J. Struct. Geol. 27, 1190-1204 (2005).

4. Crider, J. G. Oblique slip and the geometry of normal-fault linkage: mechanics and a case study from the Basin and Range in Oregon. J. Struct. Geol. 23, 1997-2009 (2001).

5. Aydin, A. \& Reches, Z. Number and orientation of fault sets in the field and in experiments. Geology. 10, 107-112 (1982).

6. Healy, D., Jones, R. R. \& Holdsworth, R. E. Three-dimensional brittle shear fracturing by tensile crack interaction. Nature. 439, 64-67 (2006).

7. Reches, Z. \& Dieterich, J. Faulting of rocks in three-dimensional strain fields. I. Failure of rocks in polyaxial, servo-control experiments. Tectonophysics. 95 , 111-132 (1983). 
8. Reches, Z. \& Lockner, D. A. Nucleation and growth of faults in brittle rocks. J. Geophys. Res. 99, 18159-18173 (1994).

9. Du,Y. \& Aydin, A. Interaction of multiple cracks and formation of echelon crack arrays. Int. J. Num. Analyt. Methods Geomech. 15, 205-218 (1991).

10. Peng, S. \& Johnson, A. M. Crack growth and faulting in cylindrical specimens of Chelmsford granite. Int. J. Rock Mech. Min. Sci. 9, 37-86 (1972).

11. Green, H. W., Scholz, C. H., Tingle, T. N., Young, T. E. \& Koczynski, T. A. Acoustic emissions produced by anticrack faulting during the olivine $\rightarrow$ spinel transformation. Geophys. Res. Lett. 19, 789-792 (1992).

12. Green, H. W., Young, T. E., Walker, D. \& Scholz, C. H. Anticrack-associated faulting at very high pressure in natural olivine. Nature. 348, 720-722 (1990).

13. Houston, H. \& Williams, Q. Fast rise times and the physical mechanism of deep earthquakes. Nature 352, 520-522 (1991).

14. Houston, H. \& Vidale, J. E. The temporal distribution of seismic radiation during deep earthquake rupture. Science. 265, 771-774 (1994).

15. Lockner, D. A., Byerlee, J. D., Kuksenko, V., Ponomarev, A. \& Sidorin, A. Quasistatic fault growth and shear fracture energy in granite. Nature 350, 39-42 (1991).

16. Thompson, B. D., Young, R. P. \& Lockner, D. A. Premonitory acoustic emissions and stick-slip in natural and smooth-faulted Westerly granite. J Geophys Res. 114, B02205J (2009).

17. Ghaffari, H. O. \& Young, R. P. Acoustic-friction networks and the evolution of precursor rupture fronts in laboratory earthquakes. Sci. Rep. 3 (2013).

18. Ben-David, O., Rubinstein, S. \& Fineberg, J. Slip-Stick: The evolution of frictional strength. Nature. 463, 76 (2010).

19. Buehler, M. \& Keten, J. S. Failure of molecules, bones, and the Earth itself. Rev Mod. Phys. 82, 1459 (2010).

20. Langer, J. S., Bouchbinder, E. \& Lookman, T. Thermodynamic theory of dislocation-mediated plasticity. Acta Materialia. 58, 3718-3732 (2010).

21. Sternlof, K. R., Rudnicki, J. W. \& Pollard, D. D. Anticrack inclusion model for compaction bands in sandstone. Journal of J. Geophys. Res. 110, (2005).

22. Heierli, J., Gumbsch, P. \& Zaiser, M. Anticrack nucleation as triggering mechanism for snow slab avalanches. Science. 321, 240-243 (2008).

23. Fletcher, R. C. \& Pollard, D. D. Anticrack model for pressure solution surfaces Geology. 9, 419-424 (1981)

24. Chang, J. C., Lockner, D. A. \& Reches, Z. Rapid acceleration leads to rapid weakening in earthquake-like laboratory experiments. Science. 338, 101-105 (2012).

25. Mogi, K. Fracture and flow of rocks under high triaxial compression. J. Geophys. Res. 76, 1255-1269 (1971)

26. Haimson, B. \& Chang, C. A new true triaxial cell for testing mechanical properties of rock, and its use to determine rock strength and deformability of Westerly granite. Int. J. Rock Mech. Min. Sci. 37, 285-296 (2000).

27. Kawasniewski, M., Xiaochun, L. \& Takhahashi, M. Ture triaxial testing of rocks (CRC Press, Taylor \& Francisco Group, Balkema, 2013).

28. Csikor, F. F., Motz, C., Weygand, D., Zaiser, M. \& Zapperi, S. Dislocation avalanches, strain bursts, and the problem of plastic forming at the micrometer scale. Science. 318, 251-254 (2007).

29. Young, R. P., Nasseri, M. H. B. \& Lombos, L. True triaxial testing of rocks (Taylor \& Francisco Group, Balkema, 2013)

30. Schubnel, A., Thompson, B. D., Fortin, J., Guéguen, Y. \& Young, R. P. Fluidinduced rupture experiment on Fontainebleau sandstone: Premonitory activity, rupture propagation, and aftershocks. Geophys. Res. Lett. 34 (2007).

31. Fortunato, S. Community detection in graphs. Phys. Rep. 486, 75-174 (2010).
32. Newman, M. E. J. \& Girvan, M. Finding and evaluating community structure in networks. Phys. Rev. E. 69, (2004).

33. Vallée, M. Source time function properties indicate a strain drop independent of earthquake depth and magnitude. Nat. Commun. 4 (2013).

34. Passelègue, F. X., Schubnel, A., Nielsen, S., Bhat, H. S. \& Madariaga, R. From SubRayleigh to Supershear Ruptures During Stick-Slip Experiments on Crustal Rocks. Science. 340, 1208-1211 (2013).

35. Schubnel, A. et al. Deep-Focus Earthquake Analogs Recorded at High Pressure and Temperature in the Laboratory. Science. 341, 1377-1380 (2013).

36. Park, J. Y. et al. High frictional anisotropy of periodic and aperiodic directions on a quasicrystal surface. Science. 309, 1354-1356 (2005).

\section{Acknowledgments}

We would like to acknowledge and thank S.D.Goodfellow (University of Toronto), M.Ghofrani Tabari, and L. Lombos for technical laboratory help and discussion on the results. The main triaxial cell for the tests was designed by L.Lombos. The authors would like to thank A.Schubnel (Laboratoire de Géologie de l'Ecole normale supérieure,France), P.Benson (University of Portsmouth), B.D.Thompson (Mine Design Engineering, Canada), P.Meredith (UCL,UK), T.Katsaga (Itasca, Toronto), K. Mair (Physics of Geological Processes, University of Oslo, Oslo, Norway), C.Marone (Pennsylvania State University, University Park, Pennsylvania, USA) and D.A.Lockner (USGS,US) for supporting the research by providing data. K.Xia and S.Huang (University of Toronto) kindly encouraged and commented on the results. We thank from Karl Peterson (University of Toronto) who helped to make the thin section images. Comments from Z.Reches (University of Oklahoma) were greatly appreciated and help improve comments and feed-backs from the research and results.

\section{Author contributions}

All authors contributed to the analysis the results and reviewed the manuscript. H.O.G.H. performed the calculations, designed the algorithms, interpreted the results and wrote the manuscript. M.H.B.N. and R.P.Y. designed the main tests. This also includes other tests that are used and included R.P.Y. design. M.H.B.N. studied micro-CT images and interpreted the thin sections. R.P.Y. supervised the research, provided the data sets and helped to analyze the results.

\section{Additional information}

Supplementary information accompanies this paper at http://www.nature.com/ scientificreports

Competing financial interests: The authors declare no competing financial interests How to cite this article: Ghaffari, H.O., Nasseri, M.H.B. \& Young, R.P. Faulting of Rocks in Three-Dimensional Stress Field by Micro-Anticracks. Sci. Rep. 4, 5011; DOI:10.1038/ srep05011 (2014).

This work is licensed under a Creative Commons Attribution-NonCommercialNoDerivs 3.0 Unported License. The images in this article are included in the article's Creative Commons license, unless indicated otherwise in the image credit; if the image is not included under the Creative Commons license, users will need to obtain permission from the license holder in order to reproduce the image. To view a copy of this license, visit http://creativecommons.org/licenses/by-nc-nd/3.0/ 